\title{
Skin conductance peaks could result from changes in vital parameters unrelated to pain
}

\author{
Abraham J. Valkenburg' ${ }^{1}$ Sjoerd P. Niehof ${ }^{2}$, Monique van Dijk', Esther J.M. Verhaar' ${ }^{1}$ and Dick Tibboel ${ }^{1}$
}

INTRODUCTION: Pain is usually assessed by the interpretation of behavior, which can be subjective. Therefore, there is an ongoing search for more objective methods. Performance of skin conductance measurement as a pain assessment tool is variable, as some studies report low specificity and a low predictive value of the method. The aim of this pilot study was to test whether autoregulation of the skin temperature influences the skin conductance of pain-free infants.

RESULTS: Skin conductance was highly correlated with skin temperature in all subjects. Moreover, a significant change in all other vital parameters was observed on comparing beforeand after-peak data.

DISCUSSION: These results indicate that sympathetic neural activity to maintain homeostasis (such as autoregulation of skin temperature) results in skin conductance peaks. Real-time evaluation of the sympathetic nervous system would be valuable for pain assessment. However, the technique should be better defined to increase both sensitivity and specificity for the measurement of pain before use in daily practice can be advocated.

METHODS: We included 11 infants, median (interquartile range $(I Q R))$ age of $34(13-76) d$, who were admitted to the surgical high-care unit for monitoring after surgery. None was treated with opioids or sedatives, and observational pain scores were low.

\section{INTRODUCTION}

Pain management and ideally pain prevention are the cornerstones of optimal postoperative care. In verbal children and adults, self-report is the generally accepted gold standard for the assessment of presence of pain. Self-report is impossible, however, in neonates and infants, intubated or sedated patients, and intellectually disabled patients. In those cases, caregivers have to rely on observational pain assessment scales such as the COMFORT-Behavior (COMFORT-B) scale (1), the Numeric Rating Scale for pain, or physiological parameters such as heart rate or blood pressure (2).

Still, researchers search for more objective methods. Recently studied methods include near-infrared spectroscopy (3), heart rate variability (4), and skin conductance measurement (5-7). The last is based on the following theory: sweat glands are sympathetically innervated and will produce sweat, and thereby salt, which increases the electrical conductance of the skin. Pain is known to stimulate sympathetic nerve activity, but animal and human studies have shown that several other physiological responses have the same effect. The primary function of the eccrine sweat glands is to enable evaporative heat loss in the autoregulation of body temperature. The following receptors and simple maneuvers influence skin sympathetic nerve traffic: temperature receptors in the central nervous system and skin, arousal and stress, respiration, cardiopulmonary receptors, sleep, and pain (8). However, Rutter (9) reports that palmar and plantar sweat glands respond more to emotional stimuli than to thermal stimuli.

Measurement of skin conductance as a pain assessment tool has been studied during heel lancing (10), intraoperatively (11), directly postoperatively (12), during postoperative care $(13,14)$, and during intensive care (15). Performances were variable, as some studies report good sensitivity (12) and others low specificity and a low predictive value (16). It could be possible that sympathetic nerve activity, other than caused by pain, influences skin conductance and, therefore, has impact on the performance of skin conductance measurement. The aim of this pilot study was to test whether autoregulation of the skin temperature influences the skin conductance of painfree infants.

\section{RESULTS}

\section{Patient Characteristics}

We included 11 subjects with a median (interquartile range (IQR)) age of $34(13-76) \mathrm{d}$. Six had had gastrointestinal surgery, three surgical closure of a congenital diaphragmatic hernia, one repair of esophageal atresia, and one a rigid bronchoscopy. Table 1 shows their baseline characteristics. Patients were studied at a median (IQR) of 5 (11-17) d after surgery. The median (IQR) COMFORT-B score before the study was 10 (9-10), indicating that none was in pain or distress.

\section{Descriptive Results}

Monitoring lasted a median (IQR) of 63 (61-65) min during which a median (IQR) number of 3,576 (3,099-3,678) samples was obtained. The median (IQR) number of skin conductance 
Table 1. Characteristics of the 11 subjects

\begin{tabular}{lc}
\hline Characteristic & Median (IQR) \\
\hline Sex (M/F) & $5 / 6$ \\
Gestational age, wk & $36(29-38)$ \\
Age, d & $34(13-76)$ \\
Postconceptional age, d & $275(259-280)$ \\
Days after surgery & $5(11-17)$ \\
Duration of measurement, min & $63(61-65)$ \\
COMFORT-B score before measurement & $10(9-10)$ \\
Skin conductance & \\
Number of peaks & $36(19-312)$ \\
Skin conductance, $\mu$ siemens & $6.42(3.13-9.21)$ \\
Number of fluctuations in skin & $0.0098(0.0047-0.0825)$ \\
Conductance per second & \\
Vital parameters & $154(130-167)$ \\
Heart rate, in beats/min & $32.19(30.54-32.88)$ \\
Skin temperature, ${ }^{\circ} \mathrm{C}$ & $0.66(0.37-1.20)$ \\
Peripheral Flow Index & $98.67(97.65-99.50)$ \\
Peripheral oxygen saturation, $\%$ & $44.37(37.39-60.85)$ \\
Respiratory rate, in breaths/min & \\
\hline COMFORT-B, COMFORT-Behavior scale; IQR, interquartile range.
\end{tabular}

peaks was 36 (19-312). The median values of the vital parameters are shown in Table 1.

Significant Differences Before and After Skin Conductance Peaks The median percentages of statistically significant differences in vital parameters before and after a skin conductance peak are displayed in Figure 1. Percentages varied from a median (IQR) of $62(59-68) \%$ for heart rate to a median (IQR) of 88 (82-94)\% for skin temperature (Friedman test: $P=0.003$ ). In one subject, peripheral oxygen saturation changed significantly after all 18 peaks (Figure 1).

\section{Correlations}

In all 11 subjects, skin temperature $\left({ }^{\circ} \mathrm{C}\right)$ was statistically significantly correlated with the skin conductance value (in $\mu$ siemens; $P$ $<0.001)$. In eight subjects, this was a positive correlation: median (IQR) Spearman's $\rho$ correlation coefficient of $0.86(0.78-0.94)$. In the remaining three subjects, it was a negative correlation: Spearman's $\rho$ correlation coefficients of $-0.48,-0.49$, and -0.99 . Background characteristics (postnatal age, type of surgery, sex, and environmental circumstances) and vital parameters of these three patients did not differ from those of the eight patients for whom a positive correlation was found.

An example of the positive correlation between skin temperature and skin conductance for one subject is displayed in Figure 2; the Spearman's $\rho$ correlation coefficient for this subject was 0.92 .

\section{DISCUSSION}

We found that in infants without evident pain, skin conductance was correlated with skin temperature. Using TrendFace ixellence

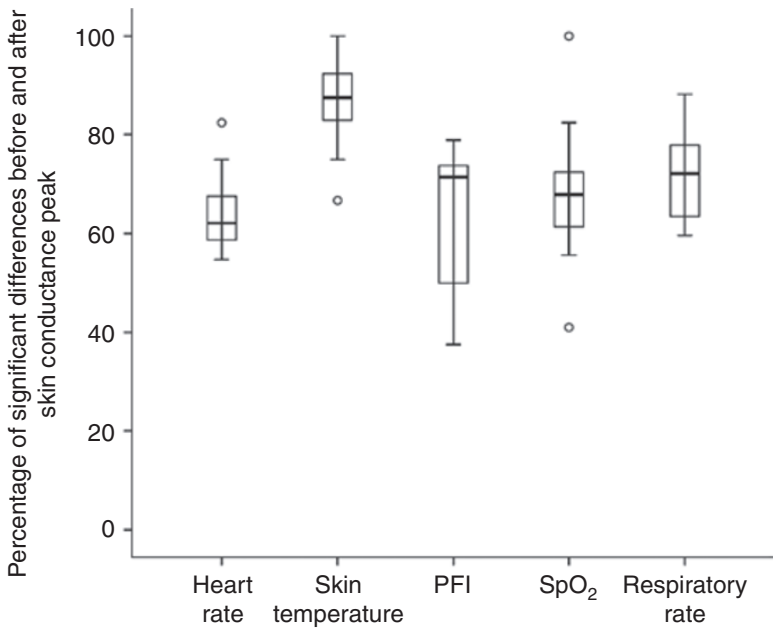

Figure 1. Percentages of significant differences before and after skin conductance peaks for the vital parameters. Median (horizontal line), 25th and 75th percentiles (box), and range (whiskers) of significant differences in vital parameters before and after a skin conductance peak. $\mathrm{PFI}$, Peripheral Flow Index; $\mathrm{SpO}_{2}$, peripheral oxygen saturation.

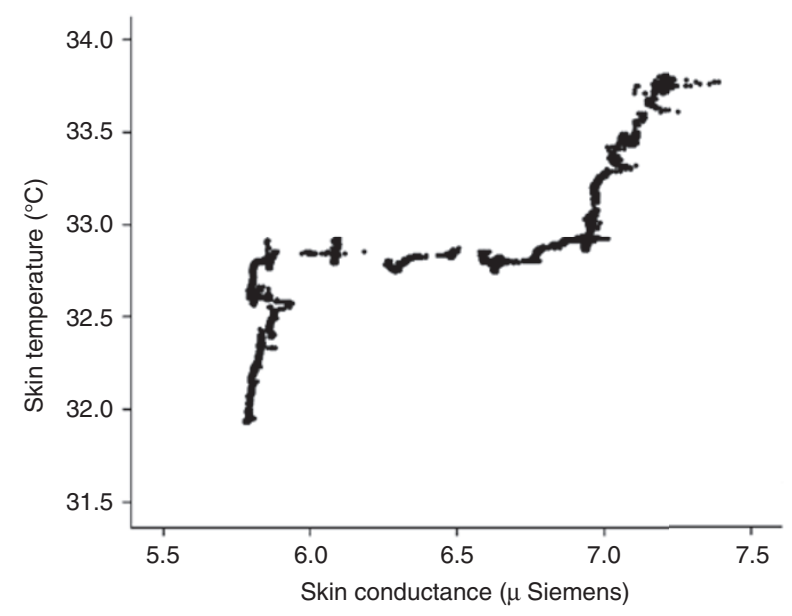

Figure 2. Correlation between skin temperature and skin conductance for subject 10. This figure is based on 2,837 paired observations (skin conductance and skin temperature) in one subject during a measurement of $1 \mathrm{~h}$. The Spearman's $\rho$ correlation coefficient is 0.92 .

(Wildau, Germany) and MATLAB MathWorks (Natick, MA) software, we were able to analyze skin conductance and vital parameters in detail, focusing on the skin conductance peaks, which represent bursts of skin sympathetic nerve activity. Vital parameters before and after a peak mostly were significantly different. For skin temperature, this was even the case in a median of $88 \%$ of the peaks. Percentages for all other four vital parameters were lower. In all 11 infants, skin temperature was highly correlated with the skin conductance. However, this pilot study does not explain why there should be both positive and negative correlations because none of the background characteristics or vital parameters differed between patients for whom a negative correlation was found and those for whom a positive correlation was found. The possibility of coincidence cannot be excluded. Our results suggest that sympathetic neural control of 
vital functions to maintain homeostasis (such as autoregulation of skin temperature) results in skin conductance peaks.

Analysis of skin sympathetic nerve activity is not a new technique. In a review article on microneurographic recordings, Wallin et al. conclude that skin sympathetic nerves are mostly involved in thermoregulation (8). Other conditions/ receptors that influence the sympathetic nerve activity are arousal, stress, respiration, sleep, cardiopulmonary receptors, and pain. Four types of skin sympathetic nerves are distinguished: vasoconstrictor, vasodilator, sudomotor, and pilomotor. Sudomotor nerves are cholinergic and cause sweat secretion (measured as a peak in skin conductance) at increased activity, for example, thermoregulation. Microneurographic recordings from the sudomotor nerves taken simultaneously with skin conductance measurement are scarce. Macefield et al. made microneurographic recordings combined with skin conductance, electrocardiogram, and skin blood flow (17). Sudomotor nerve activity appeared to be correlated with cardiac activity and skin conductance but not with skin blood flow.

Studies that evaluated skin conductance as a means of measuring (postoperative) pain reported different degrees of clinical performance $(12,16)$. For example, Choo et al. found a weak correlation between skin conductance and Numeric Rating Scale for pain in 90 postoperative children, namely 0.21 (13). They determined a cutoff value of 0.23 skin conductance peaks per $\mathrm{s}$ for the detection of severe pain (Numeric Rating Scale for pain 7 or higher) with a sensitivity of $56 \%$ and a specificity of $78 \%$. The other study in children, by Hullett et al. (12), reports a better sensitivity of $90 \%$ but a lower specificity of $64 \%$. Other relevant studies are listed in Table 2. Comparison between the studies is difficult, as cutoff points for the number of skin conductance peaks and gold standards differ between the studies. As compared with skin conductance measurement, the COMFORT-B scale performed better with a reported sensitivity of $83 \%$ and a specificity of $91 \%$ (18). Results from Loggia et al., who compared skin conductance and visual analog scale scores in healthy adults, suggest that skin conductance is not a very reliable predictor of pain (19). The authors advise caution when using autonomic measures to infer pain. The reported poor sensitivity and specificity of skin conductance for measuring (postoperative) pain could be a result of the correlation of skin conductance with, for example, autoregulation of the skin temperature, as confirmed in our study.

\section{Limitations}

None of the subjects had an arterial line for blood pressure monitoring. Therefore, we were not able to evaluate the correlation between skin conductance and blood pressure.

\section{Implications}

Preclinical and clinical studies in neonates, children, and adults are needed to make skin conductance a clinically more useful measure for postoperative pain. Preclinical studies should use microneurography as the gold standard and measure skin conductance simultaneously. These studies should not only evaluate the influence of pain and other sympathetic activity on skin conductance but also find certain rhythms, if any, in skin conductance peaks. The algorithms of the skin conductance monitor could then be made more specific for the measurement of pain by correcting for other sympathetic influences.

The clinical studies then should validate the skin conductance monitor in different age groups such as neonates, children, and adults for the measurement of (postoperative) pain (20). Furthermore, it could be difficult to compare pain-free and painful states in the same subjects and to assure stable circumstances. The clinical studies should, therefore, also integrate, for example, observational pain assessment and electroencephalography in a multimodal approach as applied by Slater et al. (21). This strategy could lead to a validated, objective pain assessment tool that can be used in clinical practice and serve as a pharmacodynamic parameter in pain research.

\section{Conclusions}

In this pilot study, we observed that, in a pain-free state, other sympathetic nerve activity, such as skin temperature autoregulation, also results in skin conductance peaks that are usually seen in pain states. Real-time evaluation of the sympathetic nervous system would be valuable for pain assessment. However, the technique should be better defined to increase both the sensitivity and specificity for the measurement of pain before use in daily practice can be advocated.

\section{METHODS}

We performed an observational pilot study in which we measured simultaneously the infants' skin conductance and vital parameters such as skin temperature, respiratory rate, heart rate, peripheral oxygen saturation, and Peripheral Flow Index.

Table 2. Studies that evaluated the performance of skin conductance as a measure of postoperative pain

\begin{tabular}{|c|c|c|c|c|c|c|}
\hline & Ledowski et al. (14) & Ledowski et al. (7) & Hullett et al. (12) & \multicolumn{2}{|c|}{ Ledowski et al. (16) } & Choo et al. (13) \\
\hline Study group & 25 adults & 75 adults & 165 children & \multicolumn{2}{|c|}{100 adults } & 90 children \\
\hline Age (years) & $21-67$ & $19-81$ & $1-16$ & \multicolumn{2}{|c|}{$18-82$} & $7-17$ \\
\hline $\begin{array}{l}\text { Cutoff value in skin } \\
\text { (conductance peaks per second) }\end{array}$ & 0.10 & 0.10 & 0.13 & \multicolumn{2}{|c|}{0.10} & 0.23 \\
\hline Gold standard & NRS (by proxy) >3 & NRS $>3$ & VAS $>3$ & NRS $>3$ & NRS $>5$ & NRS $>6$ \\
\hline Sensitivity (\%) & 89 & 89 & 90 & 50 & 58 & 56 \\
\hline Specificity (\%) & 74 & 68 & 64 & 64 & 61 & 78 \\
\hline
\end{tabular}

NRS, Numeric Rating Scale; VAS, visual analog scale. 


\section{Subjects and Setting}

The study protocol was approved by the local ethics review committee of the Erasmus University Medical Center (Rotterdam, the Netherlands). Written informed consent was obtained from all the parents. The pilot study was performed at the surgical high-care unit of Erasmus University Medical Center-Sophia Children's Hospital between July and September 2008.

Inclusion criteria were age up to and including 12 months, and being monitored in the surgical high-care unit. Exclusion criteria were mechanical ventilation, treatment with opioids or sedatives in the $24 \mathrm{~h}$ before the study, and a COMFORT-B score of 17 or higher (indicating moderate-to-severe pain or distress) before measurement.

\section{Measurements}

The infants underwent routine medical examination and nursing care in the morning. Skin conductance electrodes (see following text) were placed after nursing care, and data recording started 5-10 min later. Data were recorded for $60 \mathrm{~min}$, thereafter the electrodes were removed.

Pain and distress assessment. On the test day, the nurse who provided the morning care assessed the infant's distress and pain using the COMFORT-B scale. The COMFORT-B scale is an observational assessment tool and includes six items; each item is rated with a score from 1 to 5. Adding all six items together provides a pain rating between 6 and 30. The COMFORT-B has been validated for pain and distress assessment in critically ill and postoperative children $(1,22,23)$.

Skin conductance. Skin conductance was assessed using the Stress Detector (MedStorm, Oslo, Norway). The three pediatric skin electrodes (MedStorm) were attached to the heel of the neonate according to the manufacturer's recommendations. The software automatically defines the peaks above the amplitude threshold of $0.02 \mu$ siemens. The sample frequency of the Stress Detector is $65 \mathrm{~Hz}$. The Stress Detector records the skin conductance in $\mu$ siemens and in terms of signal quality. The other parameters, such as the number of fluctuations in skin conductance per s (NFSC) and area under the curve, are derived from the change of skin conductance over time. The NFSC was calculated over the entire measurement period $(\sim 1 \mathrm{~h})$, as this would be more precise than considering a 15- or 30-s time frame.

Vital parameters. Our surgical high-care unit uses the Philips Intellivue MP-30 monitor (Philips, Eindhoven, The Netherlands) for monitoring. The Oxisensor II neonatal probe (Covidien-Nellcor, Boulder, CO) was attached to the sole of the foot to measure the peripheral oxygen saturation and to calculate the Peripheral Flow Index. Kendall MediTrace neonatal three-lead electrocardiogram electrodes (CovidienNellcor) were used to measure heart rate and respiratory rate. The skin temperature surface probe (Philips) was attached next to the skin conductance electrodes on the heel to measure the skin temperature. During the study, the monitor was connected to a laptop computer with TrendFace software (ixellence) to record the vital parameters. Data were recorded with a sample frequency of $0.97 \mathrm{~Hz}$.

\section{Statistical Analysis}

Data extraction. The Stress Detector stores the skin conductance data in a text file with a sample frequency of $65 \mathrm{~Hz}$. The raw data as well as signal-quality data were imported in MATLAB (MathWorks, Natick, MA), a high-level technical computing language and interactive environment for algorithm development, data visualization, data analysis, and numeric computation. TrendFace software exported the vital parameter data into an Excel file with a sample frequency of $0.97 \mathrm{~Hz}$ (1.032 s). We, therefore, used skin conductance values with the same sample frequency of $0.97 \mathrm{~Hz}$.

Data analysis in MATLAB. The Stress Detector software detects peaks and hills in the skin conductance data, but does not export this information. In MATLAB, we therefore defined peaks and troughs according to the definition used in the MedStorm software, i.e., the minimal amplitude of a peak is $0.02 \mu$ siemens. The NFSC per subject were compared between the Stress Detector and the MATLAB analysis to verify the similarity between the calculations.

Because the absolute skin conductance values in $\mu$ siemens can be influenced by accumulation of sweat in the electrodes, MedStorm advises the use of the NFSC instead of the absolute skin conductance. A peak is a burst of sympathetic activity; however, peaks are not caused only by pain or distress. Mean values of vital parameters measured during $15 \mathrm{~s}$ before and after each peak were statistically compared using the $t$-test.

Data analysis in SPSS. First, we analyzed the patient characteristics using descriptive statistics. As the sample size is small in this pilot study, we report median and IQR values for the group.

Second, we analyzed the skin conductance and vital parameter data per patient using descriptive statistics. Thereafter, the mean values of the group were calculated. Also the Spearman rank order correlation coefficients between the skin conductance and vital parameters were calculated per patient.

Third, the differences in vital parameter values before and after a peak were analyzed in SPSS 19 (Chicago, IL). For each vital parameter, we calculated the percentage of values that significantly differed before and after a peak and applied the Friedman test to compare these percentages between the different vital parameters (24).

\section{ACKNOWLEDGMENT}

We thank Anne van der Eijk for her technical advice.

\section{STATEMENT OF FINANCIAL SUPPORT}

No financial assistance was received in support of this study.

\section{ADDITIONAL AUTHOR INFORMATION}

Abraham J. Valkenburg is a trainee member of Pain in Child Health, a strategic research training initiative of the Canadian Institutes of Health Research.

\section{REFERENCES}

1. van Dijk M, de Boer JB, Koot HM, Tibboel D, Passchier J, Duivenvoorden $\mathrm{HJ}$. The reliability and validity of the COMFORT scale as a postoperative pain instrument in 0 to 3 -year-old infants. Pain 2000;84: $367-77$.

2. von Baeyer CL, Spagrud LJ. Systematic review of observational (behavioral) measures of pain for children and adolescents aged 3 to 18 years. Pain 2007;127:140-50.

3. Slater R, Fitzgerald M, Meek J. Can cortical responses following noxious stimulation inform us about pain processing in neonates? Semin Perinatol 2007;31:298-302.

4. Oberlander T, Saul JP. Methodological considerations for the use of heart rate variability as a measure of pain reactivity in vulnerable infants. Clin Perinatol 2002;29:427-43.

5. Storm H. Changes in skin conductance as a tool to monitor nociceptive stimulation and pain. Curr Opin Anaesthesiol 2008;21: 796-804.

6. Hernes KG, Mørkrid L, Fremming A, Ødegården S, Martinsen ØG, Storm H. Skin conductance changes during the first year of life in full-term infants. Pediatr Res 2002;52:837-43.

7. Ledowski T, Bromilow J, Wu J, Paech MJ, Storm H, Schug SA. The assessment of postoperative pain by monitoring skin conductance: results of a prospective study. Anaesthesia 2007;62:989-93.

8. Wallin BG, Charkoudian N. Sympathetic neural control of integrated cardiovascular function: insights from measurement of human sympathetic nerve activity. Muscle Nerve 2007;36:595-614.

9. Rutter N. The dermis. Semin Neonatol 2000;5:297-302.

10. Hellerud BC, Storm H. Skin conductance and behaviour during sensory stimulation of preterm and term infants. Early Hum Dev 2002;70: 35-46.

11. Ledowski T, Bromilow J, Paech MJ, Storm H, Hacking R, Schug SA. Skin conductance monitoring compared with Bispectral Index to assess 
emergence from total i.v. anaesthesia using propofol and remifentanil. $\mathrm{Br} \mathrm{J}$ Anaesth 2006;97:817-21.

12. Hullett B, Chambers N, Preuss J, et al. Monitoring electrical skin conductance: a tool for the assessment of postoperative pain in children? Anesthesiology 2009;111:513-17.

13. Choo EK, Magruder W, Montgomery CJ, Lim J, Brant R, Ansermino JM. Skin conductance fluctuations correlate poorly with postoperative self-report pain measures in school-aged children. Anesthesiology 2010;113:175-82.

14. Ledowski T, Bromilow J, Paech MJ, Storm H, Hacking R, Schug SA. Monitoring of skin conductance to assess postoperative pain intensity. $\mathrm{Br} \mathrm{J}$ Anaesth 2006;97:862-5.

15. Gjerstad AC, Wagner K, Henrichsen T, Storm H. Skin conductance versus the modified COMFORT sedation score as a measure of discomfort in artificially ventilated children. Pediatrics 2008;122:e848-53.

16. Ledowski T, Ang B, Schmarbeck T, Rhodes J. Monitoring of sympathetic tone to assess postoperative pain: skin conductance vs surgical stress index. Anaesthesia 2009;64:727-31.

17. Macefield VG, Wallin BG. The discharge behaviour of single sympathetic neurones supplying human sweat glands. J Auton Nerv Syst 1996;61:277-86.
18. Valkenburg AJ, Boerlage AA, Ista E, Duivenvoorden HJ, Tibboel D, van Dijk M. The COMFORT-behavior scale is useful to assess pain and distress in 0- to 3-year old children with Down's syndrome. Pain 2011;152:2059-64.

19. Loggia ML, Juneau M, Bushnell MC. Autonomic responses to heat pain: Heart rate, skin conductance, and their relation to verbal ratings and stimulus intensity. Pain 2011;152:592-8.

20. Berde C, McGrath P. Pain measurement and Beecher's challenge: 50 years later. Anesthesiology 2009;111:473-4.

21. Slater R, Cornelissen L, Fabrizi L, et al. Oral sucrose as an analgesic drug for procedural pain in newborn infants: a randomised controlled trial. Lancet 2010;376:1225-32.

22. Ambuel B, Hamlett KW, Marx CM, Blumer JL. Assessing distress in pediatric intensive care environments: the COMFORT scale. J Pediatr Psychol 1992;17:95-109.

23. Ista E, van Dijk M, Tibboel D, de Hoog M. Assessment of sedation levels in pediatric intensive care patients can be improved by using the COMFORT "behavior" scale. Pediatr Crit Care Med 2005; 6:58-63.

24. Milton F. The use of ranks to avoid the assumption of normality implicit in the analysis of variance. J Am Stat Assoc 1937;32:675-701. 